https://helda.helsinki.fi

\title{
Three-dimensional splitting dynamics of giant vortices in Bose-Einstein condensates
}

\section{Räbinä, Jukka}

2018-08-22

Räbinä , J , Kuopanportti , P , Kivioja , M I , Möttönen , M \& Rossi , T 2018 , '

Three-dimensional splitting dynamics of giant vortices in Bose-Einstein condensates ',

Physical Review A, vol. 98 , no. 2 , 023624 . https://doi.org/10.1103/PhysRevA.98.023624

http://hdl.handle.net/10138/241226

https://doi.org/10.1103/PhysRevA.98.023624

cc_by

publishedVersion

Downloaded from Helda, University of Helsinki institutional repository.

This is an electronic reprint of the original article.

This reprint may differ from the original in pagination and typographic detail.

Please cite the original version. 


\title{
Three-dimensional splitting dynamics of giant vortices in Bose-Einstein condensates
}

\author{
Jukka Räbinä, ${ }^{1,{ }^{*}}$ Pekko Kuopanportti, ${ }^{2}$ Markus I. Kivioja, ${ }^{1}$ Mikko Möttönen, ${ }^{3}$ and Tuomo Rossi ${ }^{1}$ \\ ${ }^{1}$ Faculty of Information Technology, University of Jyvaskyla, P.O. Box 35, 40014 Jyvaskyla, Finland \\ ${ }^{2}$ Department of Physics, University of Helsinki, P.O. Box 43, 00014 Helsinki, Finland \\ ${ }^{3}$ QCD Labs, QTF Centre of Excellence, Department of Applied Physics, Aalto University, P.O. Box 13500, 00076 Aalto, Finland
}

(Received 20 April 2018; published 22 August 2018)

\begin{abstract}
We study the splitting dynamics of giant vortices in dilute Bose-Einstein condensates by numerically integrating the three-dimensional Gross-Pitaevskii equation in time. By taking advantage of tetrahedral tiling in the spatial discretization, we decrease the error and increase the reliability of the numerical method. An extensive survey of vortex splitting patterns is presented for different aspect ratios of the harmonic trapping potential. The discrete rotational symmetries of the splitting patterns that emerge in the time evolution are in good agreement with predictions obtained by solving the prevailing dynamical instabilities from the Bogoliubov equations. Furthermore, we observe intertwining of the split vortices in prolate condensates and a split-and-revival phenomenon in a spherical condensate.
\end{abstract}

DOI: 10.1103/PhysRevA.98.023624

\section{INTRODUCTION}

Quantized vortices are archetypal topological objects that play important roles in various branches of physics, ranging from superconductors [1] and helium superfluids [2] to cosmology [3] and astrophysics [4]. Quantized vortices exist in matter fields described by a smooth complex-valued scalar field. The essential idea is that while the complex field itself is single valued, its phase is defined only modulo $2 \pi$. Hence, the contour integral of the phase around a closed loop need not vanish, but may in fact be any integer multiple $\kappa$ of $2 \pi$. A nonzero $\kappa$ implies the presence of a quantized vortex within the loop and is referred to as the winding number of the vortex.

Bose-Einstein condensates (BECs) of atomic gases are dilute superfluids that can be described by tractable models [5,6] and controlled accurately in experiments [7]. Thus, they are excellent physical systems for studying quantized vortices. The BEC community has devoted a great deal of attention to multiquantum vortices, for which $|\kappa| \geqslant 2$, and giant vortices, for which $|\kappa| \gg 1$. Methods used to create them in gaseous BECs have so far included topological phase engineering [8-12], coherent transfer of angular momentum from photons to the atoms [13], and removal of atoms from a lattice of single-quantum vortices by a tightly focused laser beam $[14,15]$. Given that the kinetic energy of a vortex is proportional to $\kappa^{2}$, a multiquantum vortex typically has a higher energy than a cluster of $|\kappa|$ separated singly quantized vortices. This makes multiquantum vortices prone to split into singly quantized vortices. The associated instabilities and dynamics have been studied both theoretically [16-28] and experimentally [9-12]. Recent studies have also addressed utilizing vortex splitting as a means to generate quantum turbulence with controllable net circulation [29-31]. Besides being interesting due to their dynamics, multiquantum vortices

\footnotetext{
*jukka.rabina@jyu.fi
}

could also be used to implement a ballistic quantum switch [32] or realize bosonic quantum Hall states [33].

Previous theoretical studies of vortex splitting have been limited to small winding numbers $|\kappa| \leqslant 5[16-24,28]$ or to quasi-two-dimensional models pertaining to highly oblate BECs [25-27,31]. In Ref. [34], vortex splitting was studied in three dimensions up to $\kappa=45$, but only for small BECs in isotropic harmonic traps. Splitting patterns with up to tenfold rotational symmetry were observed in the numerical simulations. In this work, we carry out a more comprehensive investigation of giant-vortex splitting in three-dimensional BECs. Covering all three types of cylindrically symmetric harmonic traps (oblate, spherical, and prolate) and a wide range of repulsive interaction strengths, we simulate the temporal evolution of axisymmetric giant vortex states subjected to small random perturbations. In general, we find good agreement between the splitting patterns observed in the evolution and those predicted by linear stability analysis. Vortex splitting in prolate BECs is found to result in branched intertwining of the vortices, and spherical BECs are observed to exhibit a split-and-revival effect.

Importantly, we also find that the splitting patterns appearing in the simulated time evolution can be prone to numerical artifacts stemming from the symmetry of the underlying spatial grid. As a result, particular care should be taken when discretizing the time-dependent Gross-Pitaevskii equation (GPE) for the condensate. Specifically, the Cartesian grids used in the previous investigations tend to favor the fourfold splitting pattern, which may explain why, in Ref. [26], the highersymmetry splitting patterns predicted by the linear stability analysis were not observed to arise from random perturbations. We solve this problem by basing our time integration scheme on discrete exterior calculus [35-37] with tetrahedral tiling.

The remainder of this article is organized as follows. In Sec. II we present the time-dependent GPE, derive the Bogoliubov equations used for the linear stability analysis, and outline our numerical integration method. Section III begins 
with an analysis of the integration method and presents our numerical results. We summarize the paper in Sec. IV.

\section{THEORY AND METHODS}

\section{A. Mean-field model}

The complex-valued order parameter $\Psi$ of a dilute BEC at low temperatures obeys the time-dependent GPE

$$
i \hbar \partial_{t} \Psi(\mathbf{r}, t)=\left[-\frac{\hbar^{2}}{2 m} \nabla^{2}+V(\mathbf{r})+g|\Psi(\mathbf{r}, t)|^{2}\right] \Psi(\mathbf{r}, t),
$$

where $i$ is the imaginary unit, $\hbar$ is the reduced Planck constant, $m$ is the atom mass, and $g$ is the effective interaction strength. The order parameter is normalized such that $\int|\Psi(\mathbf{r}, t)|^{2} d^{3} r=N$ is the number of condensed atoms. We assume a cylindrically symmetric harmonic trapping potential $V(\mathbf{r})=m\left(\omega_{\rho}^{2} \rho^{2}+\omega_{z}^{2} z^{2}\right) / 2$, where $\omega_{\rho}$ and $\omega_{z}$ are the radial and axial trapping frequencies, respectively. Here and in what follows, cylindrical coordinates are denoted by $(\rho, \phi, z)$.

To have generally applicable results, we introduce dimensionless quantities by measuring position in units of the radial harmonic oscillator length $a_{\rho}=\sqrt{\hbar / m \omega_{\rho}}$, time in units of $1 / \omega_{\rho}$, the order parameter in units of $\sqrt{N} / a_{\rho}^{3 / 2}$, and the effective interaction strength in units of $a_{\rho}^{3} \hbar \omega_{\rho} / N$. Thus, the conversion to the dimensionless quantities (denoted by an overbar) is given by

$$
\overline{\mathbf{r}}=\frac{\mathbf{r}}{a_{\rho}}, \quad \bar{t}=t \omega_{\rho}, \quad \bar{\Psi}(\overline{\mathbf{r}}, \bar{t})=\Psi(\mathbf{r}, t) \frac{a_{\rho}^{3 / 2}}{\sqrt{N}}, \quad \bar{g}=\frac{g N}{a_{\rho}^{3} \hbar \omega_{\rho}} .
$$

Consequently, the dimensionless order parameter is normalized such that $\int|\bar{\Psi}(\overline{\mathbf{r}}, \bar{t})|^{2} d^{3} \bar{r}=1$, and it satisfies the dimensionless GPE

$$
i \partial_{\bar{t}} \bar{\Psi}(\overline{\mathbf{r}}, \bar{t})=\left[-\frac{1}{2} \bar{\nabla}^{2}+\bar{V}(\overline{\mathbf{r}})+\bar{g}|\bar{\Psi}(\overline{\mathbf{r}}, \bar{t})|^{2}\right] \bar{\Psi}(\overline{\mathbf{r}}, \bar{t})
$$

The dimensionless potential is given by $\bar{V}(\overline{\mathbf{r}})=\left(\bar{\rho}^{2}+\right.$ $\left.\lambda^{2} \bar{z}^{2}\right) / 2$, where $\lambda=\omega_{z} / \omega_{\rho}$ is referred to as the aspect ratio. In the cylindrical coordinates, the dimensionless Laplacian is given by $\bar{\nabla}^{2}=\partial_{\bar{\rho}}^{2}+\bar{\rho}^{-1} \partial_{\bar{\rho}}+\bar{\rho}^{-2} \partial_{\phi}^{2}+\partial_{\bar{z}}^{2}$.

Equation (1) has stationary vortex solutions $\bar{\Psi}_{\lambda, \bar{g}, \kappa}$, which depend on $\lambda, \bar{g}$, and the integer winding number $\kappa$ (we can assume $\kappa \geqslant 0$ without loss of generality). These stationary states can be written as

$$
\bar{\Psi}_{\lambda, \bar{g}, \kappa}(\overline{\mathbf{r}}, \bar{t})=f(\bar{\rho}, \bar{z}) e^{i \kappa \phi-i \bar{\mu} \bar{t}},
$$

where $f$ is a real-valued function and $\bar{\mu}$ is the dimensionless chemical potential. The stationary vortex states satisfy the time-independent equation

$$
\left[\frac{1}{2}\left(\frac{\kappa^{2}}{\bar{\rho}^{2}}-\partial_{\bar{\rho}}^{2}-\frac{1}{\bar{\rho}} \partial_{\bar{\rho}}-\partial_{\bar{z}}^{2}\right)+\bar{V}+\bar{g} f^{2}\right] f=\bar{\mu} f,
$$

which can be solved using a relaxation method [38].

\section{B. Bogoliubov equations and stability}

To study the local stability properties of a given stationary vortex solution $\bar{\Psi}_{\lambda, \bar{g}, \kappa}$, we decompose the order parameter as

$$
\bar{\Psi}(\overline{\mathbf{r}}, \bar{t})=[f(\bar{\rho}, \bar{z})+\chi(\overline{\mathbf{r}}, \bar{t})] e^{i \kappa \phi-i \bar{\mu} \bar{t}},
$$

where $\chi$ is a function describing a small perturbation such that $\int|\chi(\overline{\mathbf{r}}, \bar{t})|^{2} d^{3} \bar{r} \ll 1$. By substituting Eq. (3) into Eq. (1), neglecting the second- and third-order terms in $\chi$, and seeking oscillatory solutions of the form

$$
\chi(\mathbf{r}, \bar{t})=\sum_{q \in \mathbb{N}} \sum_{l \in \mathbb{Z}}\left[u_{q, l}(\bar{\rho}, \bar{z}) e^{i l \phi-i \bar{\omega}_{q, l} \bar{t}}+v_{q, l}^{*}(\bar{\rho}, \bar{z}) e^{i \bar{\omega}_{q, l}^{*} \bar{t}-i l \phi}\right],
$$

we obtain the Bogoliubov equations

$$
\left(\begin{array}{cc}
\mathcal{D}_{l} & \bar{g} f^{2} \\
-\bar{g} f^{2} & -\mathcal{D}_{-l}
\end{array}\right)\left(\begin{array}{c}
u_{q, l} \\
v_{q, l}
\end{array}\right)=\bar{\omega}_{q, l}\left(\begin{array}{c}
u_{q, l} \\
v_{q, l}
\end{array}\right)
$$

where the linear differential operator is defined as

$$
\mathcal{D}_{l}=\frac{1}{2}\left[\frac{(\kappa+l)^{2}}{\bar{\rho}^{2}}-\partial_{\bar{\rho}}^{2}-\frac{1}{\bar{\rho}} \partial_{\bar{\rho}}-\partial_{\bar{z}}^{2}\right]+\bar{V}+2 \bar{g} f^{2}-\bar{\mu} .
$$

The integer $l$ specifies the angular momentum of the excitation with respect to the condensate, and $q \in \mathbb{N}$ is an index for the different eigenmodes with a given $l$.

Equations (5) can be used to determine the stability characteristics of the stationary vortex state in question. If the excitation spectrum $\left\{\bar{\omega}_{q, l}\right\}$ contains at least one eigenfrequency with a positive imaginary part $\operatorname{Im}\left(\bar{\omega}_{q, l}\right)>0$, the state is dynamically unstable; otherwise, the state is dynamically stable. If the spectrum contains an excitation for which $\operatorname{Re}\left(\bar{\omega}_{q, l}\right)<0$ and $\iint\left(\left|u_{q, l}\right|^{2}-\left|v_{q, l}\right|^{2}\right) \bar{\rho} d \bar{\rho} d \bar{z} \geqslant 0$, the state is energetically unstable; if no such excitations exist, the stationary state is (locally) energetically stable. We emphasize that energetic stability is a stronger condition than dynamical stability, since the former implies the latter.

As can be observed from Eq. (4), the occupations of excitation modes with $\operatorname{Im}\left(\bar{\omega}_{q, l}\right)>0$ are predicted to increase exponentially over time; consequently, small perturbations of a dynamically unstable stationary state typically lead to large changes in its structure. For dynamically unstable multiquantum vortices, in particular, the complex-frequency modes usually induce instability against splitting of the multiply quantized vortex into singly quantized ones. In fact, the quantity $\max _{l} \max _{q} \operatorname{Im}\left(\bar{\omega}_{q, l}\right) / 2 \pi$ and the maximizing winding number $l$ can be used to predict, respectively, the inverse lifetime of a vortex and the discrete rotational symmetry of its typical splitting pattern [18]. Note, however, that the dynamically unstable modes quickly drive the system beyond the linear regime of the Bogoliubov analysis. As a result, the long-time dynamics of dynamically unstable states must be described with the time-dependent GPE (1) instead.

\section{Time integration}

Finite-difference methods have become popular for solving the time-dependent GPE because of their simplicity [39-41]. Alternative spectral methods [42-45] are also widely used. Typically, these methods rely on Cartesian spatial discretization, even though there are strong reasons to prefer simplicial grids $[46,47]$.

This work, in contrast, utilizes a time integration method based on discrete exterior calculus (DEC) [35-37], which naturally segregates the differentiable and metric structures $[48,49]$. This approach can be regarded as a generalized 
finite-difference scheme that closely resembles the finiteintegration technique [50] and the finite-difference timedomain method [51,52]. The DEC method is applicable to unstructured grids, along with being stable and conserving the particle number.

The discretization is based on a pair of interlocked threedimensional meshes: a primal (Delaunay) mesh and its dual (Voronoi) mesh. We assign to each dual node a floating-point complex number to obtain a column vector $\psi^{(k)}$ that represents the discretized order parameter at a time instant $k \Delta \bar{t} / 2$, where $k$ is an integer and $\Delta \bar{t}$ is the length of the time step. In the notation of Ref. [53], the discretized Laplacian is written as $-\star_{3} d_{2} \star_{2}^{-1} d_{2}^{T}$, where $\star_{p}$ is a diagonal matrix called the discrete Hodge star and $d_{2}$ is a sparse matrix called the discrete exterior derivative. The time integration of Eq. (1) is carried out using the central-difference method

$$
\psi^{(k+1)}=\psi^{(k-1)}-i \Delta \bar{t}\left(\frac{1}{2} \star_{3} \mathrm{~d}_{2} \star_{2}^{-1} \mathrm{~d}_{2}^{\mathrm{T}}+U^{(k)}\right) \psi^{(k)},
$$

where $U^{(k)}$ is a diagonal matrix with elements $U_{j j}^{(k)}=\left(\bar{\rho}_{j}^{2}+\right.$ $\left.\lambda^{2} \bar{z}_{j}^{2}\right) / 2+\bar{g}\left|\psi_{j}^{(k)}\right|^{2}$. Here $\bar{\rho}_{j}$ and $\bar{z}_{j}$ denote the radial and axial coordinates of the $j$ th dual node. The method is numerically stable if $\Delta \bar{t}<M^{-1}$, where $M$ is the largest diagonal element of the matrix $\frac{1}{2} \star_{3} \mathrm{~d}_{2} \star_{2}^{-1} \mathrm{~d}_{2}^{\mathrm{T}}+U^{(k)}$.

\section{RESULTS}

\section{A. Assessment of the time integrator}

First, we test our numerical integrator by propagating a stationary vortex state forward in time and investigating its stability during the simulation. We consider the normalized GPE (1) with parameters $\lambda=1, \bar{g}=300$, and $\kappa=10$. The time integrator is initialized at time instants $-\Delta \bar{t} / 2$ and 0 by setting $\psi_{j}^{(k)}=\bar{\Psi}_{\lambda, \bar{g}, k}\left(\overline{\mathbf{r}}_{j}, k \Delta \bar{t} / 2\right)$ for $k=-1,0$; here $\overline{\mathbf{r}}_{j}$ is the $j$ th dual node position of the mesh.

Let us vary the spatial mesh and consider its effects on the solution. We employ three qualitatively different grids, which correspond to Delaunay meshes generated by the node positions illustrated in Fig. 1. The simplest and most commonly used grid is the one associated with the simple cubic tiling. Its popularity is mainly based on its ease of implementation. Second, we use a body-centered-cubic (bcc) tiling [54,55], which is preferred by certain numerical studies $[46,56]$. The third option is the C15 structure, which is one of the tetrahedrally close-packed tilings [57-60]. The C15 structure has been found to be a high-quality grid for the solution of the Maxwell equations [47,53]. For each of these three grid types, we employ three discretization levels, where tasks are scaled to involve $10^{9}, 10^{10}$, or $10^{11}$ floating-point multiplications per integration over a unit time interval.
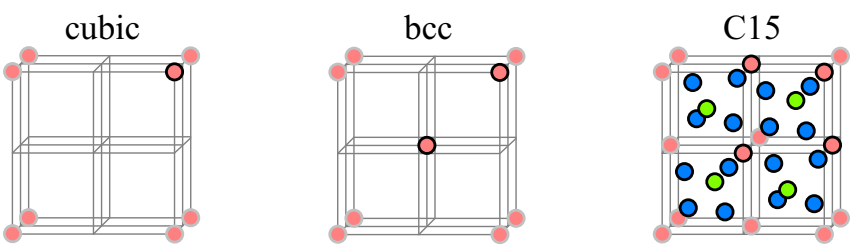

FIG. 1. Node positions for cubic, bcc, and C15 tilings.

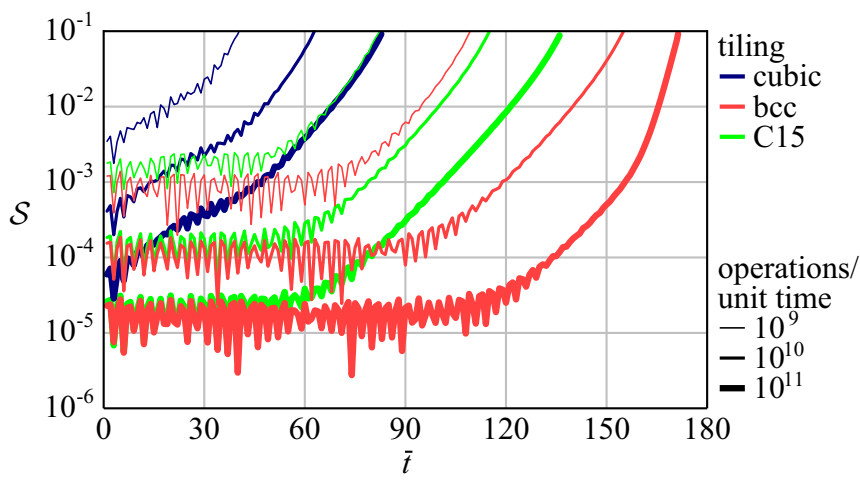

FIG. 2. Error $\mathcal{S}(\bar{t})$ induced by the numerical implementation of the GPE as a function of time for different tilings and discretization levels. The values used for the aspect ratio, interaction strength, and vortex winding number are $\lambda=1, \bar{g}=300$, and $\kappa=10$, respectively.

During the time integration, we monitor the deviation from the stationary state, $\mathcal{S}(\bar{t})=1-\left|\int \bar{\Psi}_{\lambda, \bar{g}, \kappa}^{*}(\overline{\mathbf{r}}, 0) \bar{\Psi}(\overline{\mathbf{r}}, \bar{t}) d^{3} \bar{r}\right|$, and terminate the simulation when $\mathcal{S}(\bar{t})$ exceeds 0.1 . The duration before the termination is referred to as the time span of stability. The evolution of $\mathcal{S}(\bar{t})$ is shown in Fig. 2.

The time span of stability appears to be very sensitive to the grid type used. The bcc grid offers the longest time spans because it is numerically the most isotropic of the three grids [53]. With the finest discretization level, the bcc grid leads to threefold splitting, which is the most likely physical solution for the parameter values used (see Sec. III B). In other cases, the fourfold symmetry of the cubic base grid steers the numerical solution into fourfold splitting. This demonstrates the importance of the proper tiling choice for obtaining correct physical results.

The bcc grid also offers the smallest early-stage errors before the actual vortex splitting occurs. The early-stage error is approximately proportional to $h^{4}$, where $h$ is the dual edge length. With the lowest discretization level $\left(10^{9}\right.$ operations per unit time), the average dual edge lengths are 0.20, 0.16, and 0.17 for the cubic, bcc, and $\mathrm{C} 15$ grids, respectively. The edge lengths of the finest $\left(10^{11}\right)$ and second-finest $\left(10^{10}\right)$ discretization levels are about 0.38 and 0.61 times the abovementioned edge lengths, respectively.

Owing to these results, we choose to use the bcc grid in all simulations of time evolution that follow. The Bogoliubov equations, however, can safely be solved using standard finitedifference techniques because the relevant equations (3)-(5) treat the azimuthal direction analytically.

\section{B. Dominant splitting symmetries}

Even the smallest random perturbation to a dynamically unstable stationary vortex state triggers the splitting of the vortex. To find the physically most likely splitting symmetries, the stationary vortex states $\bar{\Psi}_{\lambda, \bar{g}, \kappa}$ are perturbed by adding low-amplitude random noise in the beginning of the computation. The discretized order parameter is initialized at instants $k=-1,0$ by

$$
\psi_{j}^{(k)}=\left(1+\frac{1}{10} \zeta_{j}\right) \bar{\Psi}_{\lambda, \bar{g}, k}\left(\overline{\mathbf{r}}_{j}, k \Delta \bar{t} / 2\right)
$$




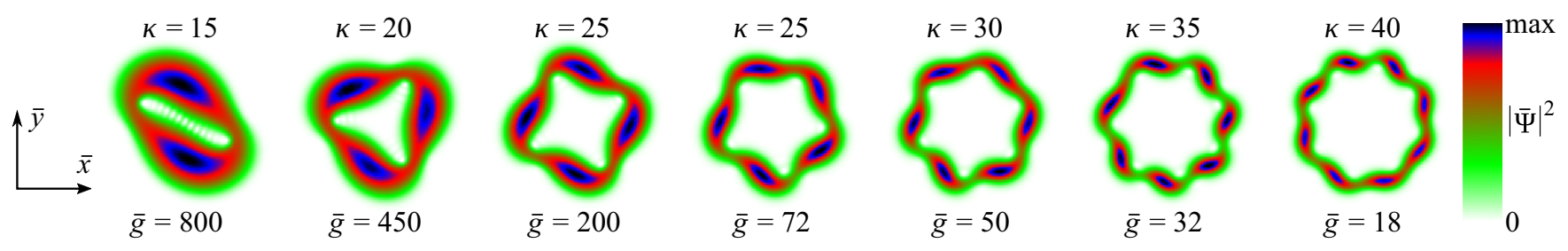

FIG. 3. Particle density $|\bar{\Psi}(\bar{t})|^{2}$ in an oblate BEC $(\lambda=10)$ integrated over $z$ at time $\min \left\{\bar{t} \mid \mathcal{P}_{l_{\mathrm{dom}}}(\bar{t})>0.2\right\}$ (see text for the definition of $\left.l_{\text {dom }}\right)$. These seven examples illustrate the typical $l_{\text {dom }}$-fold splitting patterns that appear for $l_{\text {dom }}=2,3, \ldots, 8$, respectively.

where $\zeta_{j}$ is a random variable chosen uniformly from the unit disk in the complex plane.

The spatial discretization employs a bcc grid whose dual edge lengths are less than $5 \%$ of the effective wavelength

$$
\mathcal{L}_{\lambda, \bar{g}, \kappa}=\frac{2 \pi}{\sqrt{-\int \bar{\Psi}_{\lambda, \bar{g}, \kappa}^{*}(\overline{\mathbf{r}}, 0) \bar{\nabla}^{2} \bar{\Psi}_{\lambda, \bar{g}, \kappa}(\overline{\mathbf{r}}, 0) d^{3} \bar{r}}} .
$$

This corresponds to the second-finest discretization level of Sec. III A. The computational domain is a rectangle that contains all points $\overline{\mathbf{r}}$ for which $\left|\bar{\Psi}_{\lambda, \bar{g}, \kappa}(\overline{\mathbf{r}}, 0)\right|$ is greater than $10^{-5}$ times its maximum value. Zero particle density is used as the boundary condition.

The following procedure is applied to find dominant splitting symmetries. During a time integration, splitting indicators $\mathcal{P}_{l}(\bar{t})=\left.\left|\int e^{i l \phi}\right| \bar{\Psi}(\overline{\mathbf{r}}, \bar{t})\right|^{2} d^{3} \bar{r} \mid$ are computed at each time instant $\bar{t}$. The number $l_{\text {dom }} \in \mathbb{N}_{+}$, for which $\mathcal{P}_{l_{\text {dom }}}(\bar{t}) \geqslant \mathcal{P}_{l}(\bar{t}) \forall l \in$ $\mathbb{N}_{+}$, indicates the dominant splitting symmetry. Vortex dynamics is divided into three categories: If $\mathcal{P}_{l_{\mathrm{dom}}}$ exceeds 0.1 before the time reaches 200 , we classify the case as vortex splitting with $l_{\mathrm{dom}}$-fold symmetry (see Fig. 3). Otherwise, if $\mathcal{S}(\bar{t})<0.1$ for the entire integration interval $0 \leqslant \bar{t} \leqslant 200$, we detect a relatively stable vortex and label this case as "no split". Otherwise, we observe an unstable vortex without any obvious dominant splitting symmetry; this case is called "unclear".

We use three different values for the aspect ratio $\lambda$ to represent oblate $(\lambda=10)$, spherical $(\lambda=1)$, and prolate $(\lambda=0.1)$ condensates. In addition, we vary the effective interaction strength $\bar{g}$ and the winding number $\kappa$ to obtain a comprehensive understanding of the splitting process. The resulting splittingsymmetry diagrams are presented in Fig. 4 . We note that the observations from the time integrator are not entirely unique, since the results depend slightly on the seed of the random number generator. To reduce variation, we have simulated each splitting process twice with different seeds and chosen the splitting symmetry that is closer to the prediction of the Bogoliubov equations. Here the Bogoliubov prediction is defined as the rotational symmetry corresponding to the value of $|l|$ for which $\max _{q} \operatorname{Im}\left(\bar{\omega}_{q, l}\right)$ is largest [26]. Visual inspection of Fig. 4 shows that the results of the time integration mostly coincide with the Bogoliubov predictions.

The characteristics of the splitting symmetries as functions of $\bar{g}$ and $\kappa$ are similar for different aspect ratios $\lambda$. For each of the three $\lambda$ values considered in Fig. 4, rotational symmetries of orders from 2 to 9 are observed in the splitting of vortices with $2 \leqslant \kappa \leqslant 40$. When the aspect ratio is decreased, a given splitting symmetry is found at higher interaction strengths, which is explained by the increased size of the condensate. The

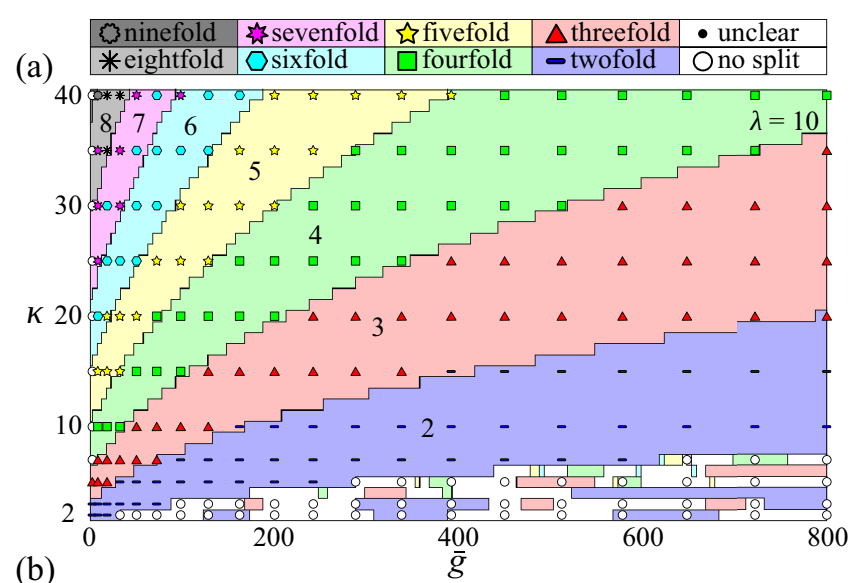

(b)

$\bar{g}$

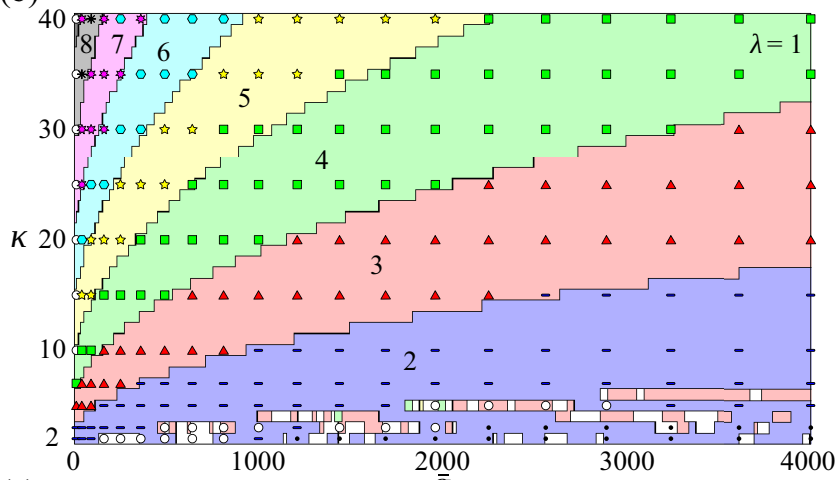

(c)

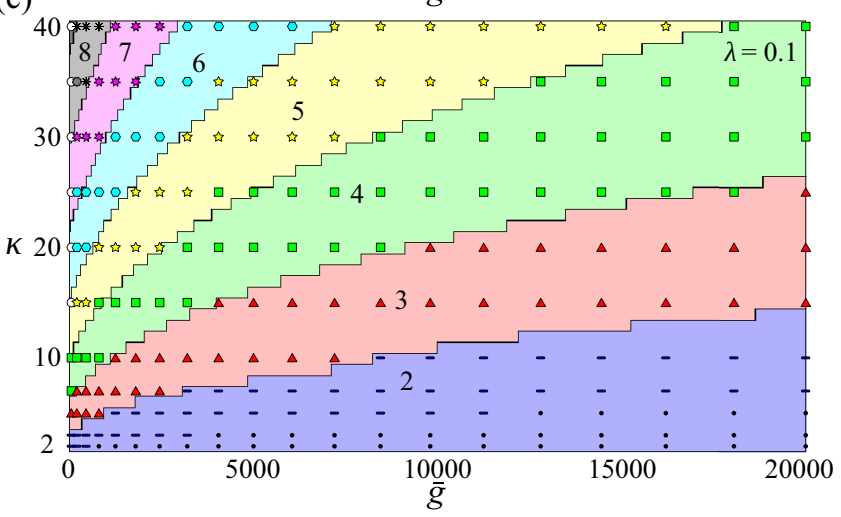

FIG. 4. Observed splitting symmetries plotted in the plane of the effective interaction strength $\bar{g}$ and the winding number $\kappa$ in (a) oblate $(\lambda=10)$, (b) spherical $(\lambda=1)$, and (c) prolate $(\lambda=0.1)$ condensates. The symbol indicates the result of the time integration, while the background color (shade) corresponds to the prediction of the Bogoliubov equations, namely, the value of $|l|$ for which $\max _{q} \operatorname{Im}\left(\bar{\omega}_{q, l}\right)$ is largest. These values are also given by the numbers inside the larger regions. 
(a)

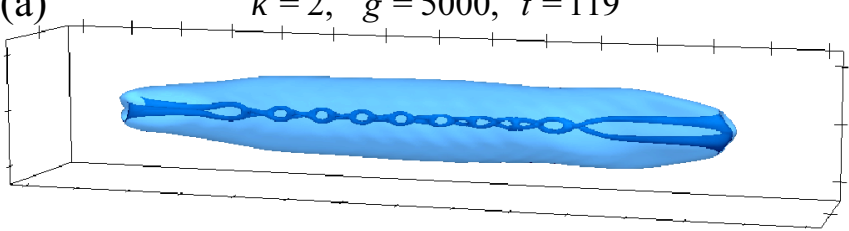

(b)

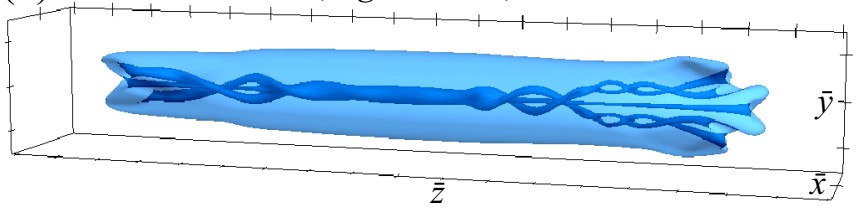

FIG. 5. Transparent isosurface of the particle density $|\bar{\Psi}(\bar{t})|^{2}$ demonstrates the intertwining of (a) two- and (b) five-quantum vortices in a prolate condensate $(\lambda=0.1)$. The domain dimensions are $13 \times 13 \times 73$ and $15 \times 15 \times 91$, respectively.

most significant qualitative difference between the three panels in Fig. 4 is that the unclear splitting symmetries appear only in the spherical and prolate condensates. This phenomenon will be studied in more detail in the next section.

\section{Intertwining of vortices}

In prolate condensates, we observe vortices to intertwine as they split, as illustrated in Fig. 5. Similar intertwining processes of doubly quantized vortices $(\kappa=2)$ have already been discovered in Refs. [18,20,21]. Our study demonstrates

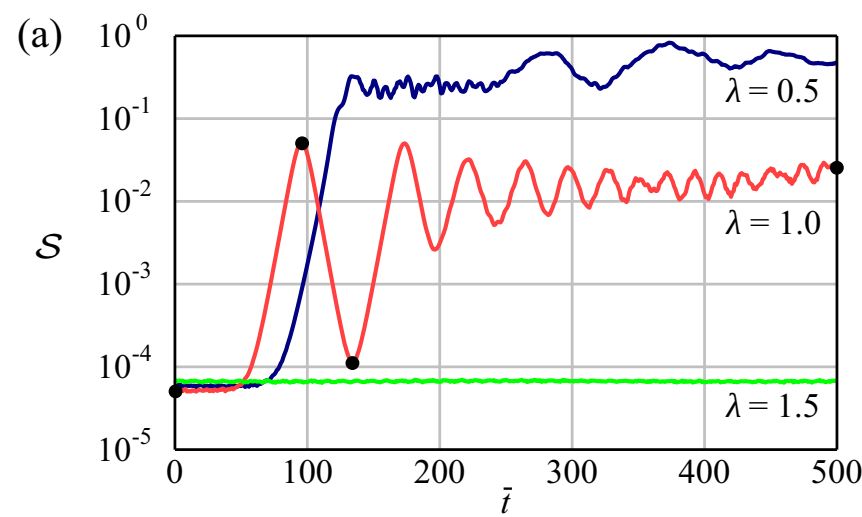

(b)

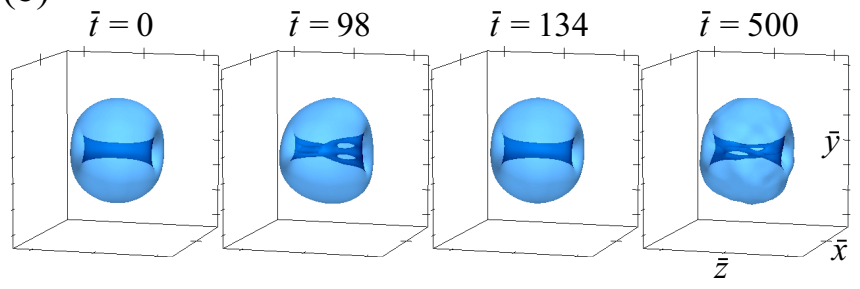

FIG. 6. Effect of the aspect ratio $\lambda$ on the stability of a threequantum vortex with the interaction strength set to $\bar{g}=1000 / \lambda$. (a) The deviation $\mathcal{S}(\bar{t})$ from the stationary state as a function of time. (b) Particle density isosurfaces visualizing the split-and-revival effect observed for $\lambda=1.0$. The corresponding stationary state has $\max _{l} \max _{q} \operatorname{Im}\left(\bar{\omega}_{q, l}\right) \approx 0.101$, with the maximum occurring for $|l|=2$. The domain dimensions are $14 \times 14 \times 13$. The states shown in (b) are marked with black dots in (a).

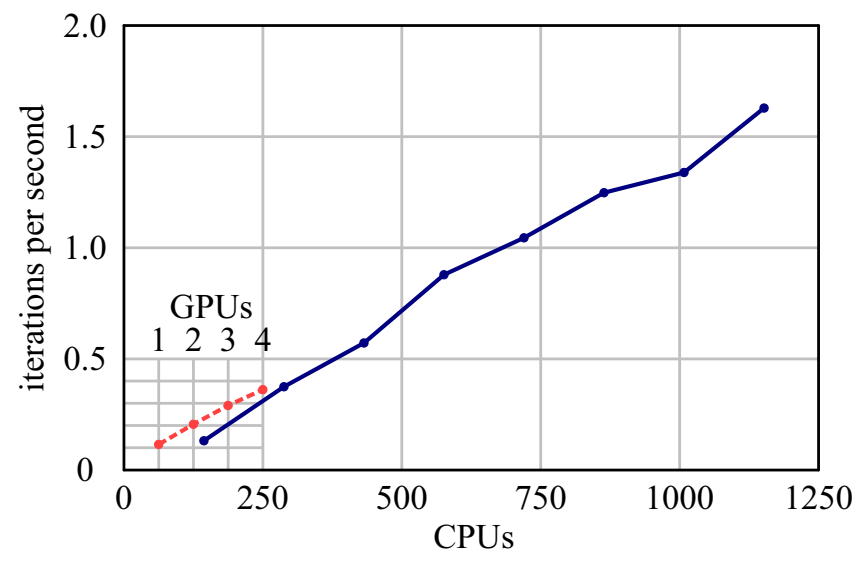

FIG. 7. Performance of the CPU implementation (solid blue line) and the GPU implementation (dashed red line) as a function of the computing resources. One iteration corresponds to an integration over one unit of time.

that intertwining also occurs for large winding numbers. Figure 5(b) shows the intertwining of a five-quantum vortex $(\kappa=5)$ proceeding in a branched fashion.

The intertwining of vortices does not take place in the oblate condensates with the aspect ratio $\lambda=10$, but the phenomenon seems to emerge when $\lambda$ is close to 1 . To investigate this further, we consider the dynamics of three-quantum vortices $(\kappa=3)$ for different intermediate aspect ratios. To equalize the local peak interaction strengths, the effective interaction strength $\bar{g}$ is chosen to be inversely proportional to the aspect ratio by setting $\bar{g}=1000 / \lambda$. The results of these simulations are presented in Fig. 6.

The simulations indicate that the three-quantum vortices are stable in oblate condensates with $\lambda \geqslant 1.5$. In prolate condensates with $\lambda \leqslant 0.5$, the vortices seem to be unstable and exhibit intertwining. In the regime $0.5<\lambda<1.5$, no prevalent behavior is detected. Nevertheless, in a condensate with $\lambda=1.0$, we discover a cyclic splitting process, where the vortex begins to split but then returns nearly to its initial state. This split-and-revival effect is illustrated in Fig. 6(b).

\section{Computational performance}

The time integrations of this paper were executed on central processing units (CPUs), but we have also implemented the solver with graphics processing units (GPUs). The performances of the two implementations are studied here by measuring the simulation times in the case $\lambda=0.1, \bar{g}=5000$, and $\kappa=20$. We utilize up to 9612 -core Intel Xeon CPUs (E5-2690 v3, Haswell-EP, 64 bits) and up to four NVIDIA Tesla P100 GPUs. The results in Fig. 7 indicate that the performance of the GPU implementation on one GPU corresponds to the performance of the CPU implementation executed on at least $60 \mathrm{CPU}$ cores.

\section{CONCLUSION}

In summary, we have studied the splitting dynamics of giant vortices in dilute BECs, with particular emphasis on the time integration of the three-dimensional GPE. We showed that a significant reduction of the numerical error is achieved when a tetrahedral spatial tiling is used instead of the customary 
Cartesian grid. Importantly, the careful choice of the numerical method provided us with the physically correct splitting symmetry.

Comprehensive maps of vortex splitting symmetries were presented for oblate, spherical, and prolate BECs. The solutions obtained by time integration were found to agree with the linear stability analysis based on the Bogoliubov equations. Splitting patterns exhibiting up to ninefold rotational symmetry were obtained with both approaches, the highest-order symmetries occurring for small interaction strengths and large winding numbers.

The splitting-induced intertwining of giant vortices in prolate condensates was demonstrated and a branched variant of the process was detected. The aspect ratios for which the intertwining became observable were also assessed. A splitand-revival phenomenon, where the vortex almost returned to its initial state after splitting temporarily, was observed in the crossover from a dynamically stable vortex into an unstable one as a function of the aspect ratio.
The performance study presented in Sec. IIID indicates nearly optimal scalability of the CPU implementation and promising performance for the GPU implementation. A future study of how the GPU performance scales with a larger number of GPUs would help us accomplish more challenging tasks than is currently possible with CPUs. These tasks may include solving the dynamics of a lattice of monopole-antimonopole pairs $[61,62]$.

\section{ACKNOWLEDGMENTS}

We have received funding from the European Research Council under Consolidator Grant No. 681311 (QUESS), the Technology Industries of Finland Centennial Foundation, and the Academy of Finland through its Centres of Excellence Program (Projects No. 312300, No. 251748, and No. 284621) and Grant No. 308632. Computing resources were provided by the CSC - IT Center for Science, which is owned by the Finnish Ministry of Education and Culture.
[1] Superconductivity, edited by R. D. Parks (Dekker, New York, 1969).

[2] R. J. Donnelly, Quantized Vortices in Helium II (Cambridge University Press, Cambridge, 1991).

[3] A. Vilenkin and E. P. S. Shellard, Cosmic Strings and Other Topological Defects (Cambridge University Press, Cambridge, 1994).

[4] P. W. Anderson and N. Itoh, Nature (London) 256, 25 (1975).

[5] F. Dalfovo, S. Giorgini, L. P. Pitaevskii, and S. Stringari, Rev. Mod. Phys. 71, 463 (1999).

[6] A. L. Fetter, Rev. Mod. Phys. 81, 647 (2009).

[7] B. P. Anderson, J. Low Temp. Phys. 161, 574 (2010).

[8] A. E. Leanhardt, A. Görlitz, A. P. Chikkatur, D. Kielpinski, Y. Shin, D. E. Pritchard, and W. Ketterle, Phys. Rev. Lett. 89, 190403 (2002).

[9] Y. Shin, M. Saba, M. Vengalattore, T. A. Pasquini, C. Sanner, A. E. Leanhardt, M. Prentiss, D. E. Pritchard, and W. Ketterle, Phys. Rev. Lett. 93, 160406 (2004).

[10] T. Isoshima, M. Okano, H. Yasuda, K. Kasa, J. A. M. Huhtamäki, M. Kumakura, and Y. Takahashi, Phys. Rev. Lett. 99, 200403 (2007).

[11] T. Kuwamoto, H. Usuda, S. Tojo, and T. Hirano, J. Phys. Soc. Jpn. 79, 034004 (2010).

[12] H. Shibayama, Y. Yasaku, and T. Kuwamoto, J. Phys. B 44, 075302 (2011).

[13] M. F. Andersen, C. Ryu, P. Cladé, V. Natarajan, A. Vaziri, K. Helmerson, and W. D. Phillips, Phys. Rev. Lett. 97, 170406 (2006).

[14] P. Engels, I. Coddington, P. C. Haljan, V. Schweikhard, and E. A. Cornell, Phys. Rev. Lett. 90, 170405 (2003).

[15] T. P. Simula, P. Engels, I. Coddington, V. Schweikhard, E. A. Cornell, and R. J. Ballagh, Phys. Rev. Lett. 94, 080404 (2005).

[16] H. Pu, C. K. Law, J. H. Eberly, and N. P. Bigelow, Phys. Rev. A 59, 1533 (1999).

[17] T. P. Simula, S. M. M. Virtanen, and M. M. Salomaa, Phys. Rev. A 65, 033614 (2002).

[18] M. Möttönen, T. Mizushima, T. Isoshima, M. M. Salomaa, and K. Machida, Phys. Rev. A 68, 023611 (2003).
[19] Y. Kawaguchi and T. Ohmi, Phys. Rev. A 70, 043610 (2004).

[20] J. A. M. Huhtamäki, M. Möttönen, T. Isoshima, V. Pietilä, and S. M. M. Virtanen, Phys. Rev. Lett. 97, 110406 (2006).

[21] A. Muñoz Mateo and V. Delgado, Phys. Rev. Lett. 97, 180409 (2006).

[22] K. Gawryluk, M. Brewczyk, and K. Rzążewski, J. Phys. B 39, L225 (2006).

[23] E. Lundh and H. M. Nilsen, Phys. Rev. A 74, 063620 (2006).

[24] T. Karpiuk, M. Brewczyk, M. Gajda, and K. Rzążewski, J. Phys. B 42, 095301 (2009).

[25] P. Kuopanportti, E. Lundh, J. A. M. Huhtamäki, V. Pietilä, and M. Möttönen, Phys. Rev. A 81, 023603 (2010).

[26] P. Kuopanportti and M. Möttönen, Phys. Rev. A 81, 033627 (2010).

[27] P. Kuopanportti and M. Möttönen, J. Low Temp. Phys. 161, 561 (2010).

[28] A. Cidrim, A. C. White, A. J. Allen, V. S. Bagnato, and C. F. Barenghi, Phys. Rev. A 96, 023617 (2017).

[29] M. Abraham, I. Aranson, and B. Galanti, Phys. Rev. B 52, R7018 (1995).

[30] I. Aranson and V. Steinberg, Phys. Rev. B 53, 75 (1996).

[31] A. Cidrim, F. E. A. dos Santos, L. Galantucci, V. S. Bagnato, and C. F. Barenghi, Phys. Rev. A 93, 033651 (2016).

[32] A. S. Mel'nikov and V. M. Vinokur, Nature (London) 415, 60 (2002).

[33] M. Roncaglia, M. Rizzi, and J. Dalibard, Sci. Rep. 1, 43 (2011).

[34] J. Li, D.-S. Wang, Z.-Y. Wu, Y.-M. Yu, and W.-M. Liu, Phys. Rev. A 86, 023628 (2012).

[35] A. N. Hirani, Discrete exterior calculus, Dissertation (Ph.D.), California Institute of Technology, 2003.

[36] M. Desbrun, E. Kanso, and Y. Tong, in Discrete Differential Geometry, edited by A. I. Bobenko, J. M. Sullivan, P. Schröder, and G. M. Ziegler (Birkhäuser, Basel, 2008), pp. 287-324.

[37] A. Stern, Y. Tong, M. Desbrun, and J. E. Marsden, in Geometry, Mechanics, and Dynamics, edited by D. E. Chang, D. D. Holm, G. Patrick, and T. Ratiu (Springer, New York, 2015), pp. 437-475. 
[38] J. M. Ortega and W. C. Rheinboldt, Iterative Solution of Nonlinear Equations in Several Variables (Academic Press, New York, 1970).

[39] M. M. Cerimele, M. L. Chiofalo, F. Pistella, S. Succi, and M. P. Tosi, Phys. Rev. E 62, 1382 (2000).

[40] F. I. Moxley, T. Byrnes, B. Ma, Y. Yan, and W. Dai, J. Comput. Phys. 282, 303 (2015).

[41] L. E. Young-S, D. Vudragović, P. Muruganandam, S. K. Adhikari, and A. Balaž, Comput. Phys. Commun. 204, 209 (2016).

[42] W. Bao, D. Jaksch, and P. A. Markowich, J. Comput. Phys. 187, 318 (2003).

[43] W. Bao and H. Wang, J. Comput. Phys. 217, 612 (2006).

[44] X. Antoine, W. Bao, and C. Besse, Comput. Phys. Commun. 184, 2621 (2013).

[45] C. M. Dion and E. Cancès, Phys. Rev. E 67, 046706 (2003).

[46] A. Bossavit, in Scientific Computing in Electrical Engineering, edited by W. H. A. Schilders, E. J. W. ter Maten, and S. H. M. J. Houben (Springer, Berlin, 2004), pp. 128-136.

[47] J. Räbinä, S. Mönkölä, and T. Rossi, SIAM J. Sci. Comput. 37, B834 (2015).

[48] A. Bossavit and L. Kettunen, Int. J. Numer. Model. 12, 129 (1999).

[49] A. Bossavit and L. Kettunen, IEEE Trans. Magn. 36, 861 (2000).
[50] T. Weiland, in Computational Electromagnetics, edited by P. Monk, C. Carstensen, S. Funken, W. Hackbusch, and R. H. W. Hoppe (Springer, Berlin, 2003), pp. 183-198.

[51] K. S. Yee, IEEE Trans. Antennas Propag. 14, 302 (1966).

[52] A. Taflove, IEEE Trans. Electromagn. Compat. 22, 191 (1980).

[53] J. Räbinä, L. Kettunen, S. Mönkölä, and T. Rossi, ESAIM: Math. Model. Numer. Anal., https://doi.org/10.1051/m2an/2018017.

[54] D. M. Y. Sommerville, Proc. Edinburgh Math. Soc. 41, 49 (1922).

[55] J. Conway and N. J. A. Sloane, Sphere Packings, Lattices and Groups, 3rd ed. (Springer, New York, 1999).

[56] E. VanderZee, A. N. Hirani, and D. Guoy, in Proceedings of the 17th International Meshing Roundtable, edited by R. V. Garimella (Springer, Berlin, 2008), pp. 19-35.

[57] J. B. Friauf, J. Am. Chem. Soc. 49, 3107 (1927).

[58] P. Paufler, Intermetallics 19, 599 (2011).

[59] J. M. Sullivan, in Foams and Emulsions, edited by J. F. Sadoc and N. Rivier (Springer, Dordrecht, 1999), pp. 379-402.

[60] D. Eppstein, J. M. Sullivan, and A. Üngör, Comput. Geom. 27, 237 (2004).

[61] M. W. Ray, E. Ruokokoski, S. Kandel, M. Möttönen, and D. S. Hall, Nature (London) 505, 657 (2014).

[62] M. W. Ray, E. Ruokokoski, K. Tiurev, M. Möttönen, and D. S. Hall, Science 348, 544 (2015). 